\title{
Lossless Quality Steganographic Color Image Compression
}

\author{
Tamer Rabie \\ Associate Professor of Computer Engineering \\ Department of Electrical \& Computer Engineering \\ University of Sharjah, UAE
}

\begin{abstract}
This paper develops a steganography-based paradigm for lossless-quality compression of high-resolution color images acquired by megapixel cameras. Our scheme combines space-domain and frequency-domain image processing operations where in the space domain, color-brightness separation is exploited, and in the frequency domain, spectral properties of the Fourier magnitude and phase of the color image is exploited. Working in both domains concurrently allows for an approach to ultrahigh-resolution image compression that addresses both issues of quality and storage size. Experimental results as well as empirical observations show that our technique exceeds the highest quality JPEG image compression standard in terms of compression rates while being very competitive with JPEG in the overall fidelity of the decompressed image, with the added advantage of being able to recover the original fine details in the color image without any degradations common in lossy image compression techniques.
\end{abstract}

Keywords-Lossless Quality Compression; Steganography; Color Image Compression; Lab color space; RGB color space, Frequency Domain Data Hiding

\section{INTRODUCTION}

The most widely used compression technique for storing color images acquired by high-resolution digital cameras is the Joint Photographic Experts Group (JPEG) image format. Needless to say, images compressed using JPEG's lossy compression paradigm suffer from JPEG blocking artifacts due to the nature of the lossy Discrete Cosine Transform (DCT) $8 \times 8$ block-size used by the JPEG compression standard, as well as the inherent loss of fine details due to degredation in picture resolution. Figure 1 shows an example of typical JPEG blocking artifacts appearing in images compressed by this standard. High compression rates ${ }^{1}$ result in images with blockyness in the blue and red channels. This is the reason most professional digital cameras implement a RAW image storage format which leaves the image uncompressed and huge.

Attempts to remove these blocking artifacts from JPEGcompressed images have been dicussed extensively in the literature [38], [19], [21], [34]. Nevertheless, these techniques fail to retain the high quality of fine details in the compressed images which is an inevitable consequence of the JPEG compression scheme. As a matter of fact, while deblocking the JPEG-compressed images, these methods remove more of the fine detail in the process [22].

\footnotetext{
${ }^{1}$ Compression Rate $=1-($ compressed file size/uncompressed file size $)$
}

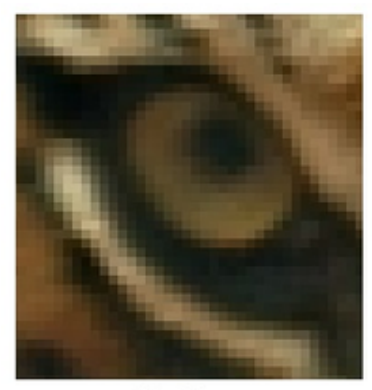

original image

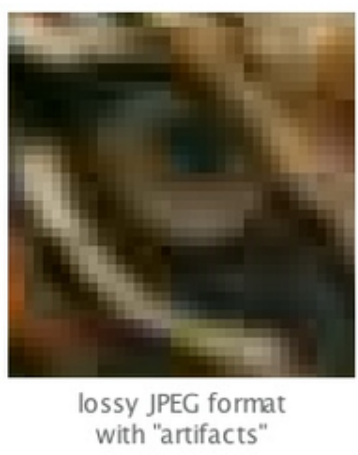

Fig. 1: JPEG compressed color image showing blocking artifacts in the right image portion.

This paper presents a detailed account of an improved color image compression scheme that is lossless in the luminance image quality while being lossy only in the chrominance channel representations, which have an insignificant effect on the overall decompression quality as we will show. This new scheme provides important improvements over the original Fourier-domain image-hiding compression framework, first presented in [32], which suffered from discoloration artifacts in the decompressed color image due to the nature of the Frequency domain color embedding and extraction scheme used.

The proposed idea is to utilize the space-domain and Fast Fourier Transform (FFT) domain of the high-resolution color image to enhance the compression rate while avoiding the undesirable effects of reduced detail quality and blocking artifacts inherent in other commonly used image compression schemes. In the space domain, image color-brightness separation is exploited, and in the FFT domain, spectral properties of the Fourier magnitude and phase of the acquired image is exploited. Working in both domains concurrently allows us to address both issues of quality and storage size when dealing with high-resolution color image compression.

Information hiding techniques, commonly known as steganography when dealing with hiding secret messages into a cover medium to form a "stego" medium [28], or watermarking when copyright protection of multimedia data is involved [37], have received a great deal of attention in the past decade [4], [35], [17], [20], [23]. Motivated by growing concern about the protection of intellectual property on the Internet and by 
the threat of a ban for encryption technology, the interest in information hiding systems has been increasing over the years [27].

Techniques for information hiding inside digital images have been generally confined to three popular approaches, namely; the spatial approach, which involve manipulation of the least significant bit (LSB) of an image pixel value and the rearrangement of image colors to create LSB or parity bit patterns, which correspond to the message being hidden [3], [12], [6]; the compression approach [8], [5]; and the frequency domain approach [7], [30], with variants that try to improve four different aspects; perceptibility, capacity, security, and robustness [11].

Perceptibility deals with the amount of "distortion" in the cover medium due to embedding information and if this information will lead to a visibly (visually or audibly) unacceptable level of the cover medium. Capacity refers to the amount of information that can be hidden in the cover medium relative to the change in perceptibility. For images, capacity is measured in bits per pixel. Security refers to an eavesdropper's inability to detect and inturn extract or change the hidden information, and robustness to the amount of modification the stego medium can withstand before an adversary can destroy the hidden information.

The main driving force behind data hiding in images is the fact that most images have inter-pixel relations that vary between high correlation and almost no correlation. The idea is to identify the redundancy in the pixel information of the cover image where the correlation is the least and use it to embed the secret information that we seek to hide.

The rest of this paper is organized as follows. In section II we present prior work in the area of steganography-based highcompression rate techniques. Section III discusses the theory behind separation of phase from magnitude in the frequencydomain of the image, while section IV briefly reviews the different color standards commonly used in image processing operations and discusses the advantage of space-domain colorbrightness separation for preservation of fine details during image compression. Our proposed high-fidelity Losslessquality Steganography-based color image compression scheme which we denote as "LqSteg" is discussed in section V, and section VI presents our results and comparisons to the popular JPEG compression standard, and also demonstrates the highest image quality that can be achieved based on our approach. Finally, concluding remarks appear in section VII.

\section{PREVIOUS WORK}

Steganography has been extensively used for hiding secret data into different media types and many schemes have been proposed in the literature which try to address one or more important aspects, namely; perceptibility, capacity, security, and robustness, but rarely have they been utilized effectively for lossless-quality image compression. The only accounts of published work in the area of image compression that utilize steganography techniques (other than the preliminary idea first presented by the author in [32]) are; the compressive data hiding scheme proposed by Campisi et. at. [1], the dual domain watermarking for authentication and compression method presented in [40], the DCT-based data-hiding method to embed color information in JPEG grey level images published in [10], the Semantic compression method for greyscale image compression proposed by Zhang \& Zhang [39], and the Reversible Data Hiding-based compression Technique proposed by Kang et. al [18].

In [1] the chrominance information is subsampled and embedded in the discrete wavelet transform (DWT) domain of the luminance component. This method was used as a preprocessing stage to improve the performance of popular image compression schemes that are optimized for grayscale image compression. In their scheme the color image is converted to the YIQ color space where the chrominance components are processed in the DWT domain and embedded in the wavelet domain of the luminance component of the original color image, with compression rates reaching $98 \%$. The method, however, differs completely from our approach in the fact that it is highly lossless and is not concerned with maintaining highfrequency image structure, and therefore, we cannot compare our scheme to their results because of the different objectives of the two methods.

The dual domain watermarking and compression scheme proposed in [40] is an extension of the previous method in [1] where they implement the watermarking as a DCT-DWT dual domain algorithm and apply it for the protection and compression of cultural heritage imagery, with results that also fail to preserve the high-frequency image structure in the original image.

In [10], the objective of their scheme is to allow free access to compressed grey-level images and give color image access only if the user owns a secret key. This method consists of color quantization, color ordering, and DCT-based data hiding to embed the color information of the image in the corresponding compressed grey-level image.

In [39] the authors create a compact image from the full size grey-scale image by downsampling the pixels in the original image, and collect the estimation errors between the downsampled compact image pixels and the original image pixels. Then, the estimation errors, which provide the compression information, are embedded into the compact image using a LSB replacement technique to produce a compressed image with smaller size and similar content. The maximum compression rates they have been able to achieve for greyscale images was $50 \%$.

In [18] the authors implement a lossless compression technique for further compressing JPEG compressed images by hiding some of the image data inside the entropy coded portion of the JPEG quantized DCT coefficients. The maximum compression rates they have been able to achieve for grey-scale images was $0.36 \%$ above the already compressed JPEG image.

Another Scheme proposed by Qin et. al. [29] is the Joint Data-Hiding and Compression technique which combines the two functions of data-hiding and commpression simultaneously. It should be noted, however, that this technique does not fall into the same category of Steganography-based compression techniques since the authors do not use data hiding for the purpose of compression, but rather integrate both functions of data hiding and compression in one process. 


\section{SignificAnCE OF MAgnitude AND Phase}

It has been experimentally established that for many images, the phase of the Fourier transform is more important than the magnitude [15], [24], [25]. Specifically if

$$
F(u, v)=|F(u, v)| e^{j \theta(u, v)}
$$

denotes the two-dimensional (2D) Fourier transform of an image $f(x, y)$, then the inverse Fourier transform of $e^{j \theta(u, v)}$ has many recognizable features in common with the original, whereas the inverse Fourier transform of $|F(u, v)|$ generally bears no resemblance to the original. This is illustrated in figure 2 where figure 2-(a) is a Red, Green, Blue (RGB) color image and figure 2-(b) is the phase-only image, i.e., the inverse Fourier transform of $e^{j \theta(u, v)}$. Clearly, the phase-only image retains many of the features of the original. By contrast, the magnitude-only image, i.e., the inverse Fourier transform of $|F(u, v)|$, shown in figure 2-(c), bears no resemblance to the original image. As is evident in this example, the phaseonly image often has the general appearance of a high-pass filtered version of the original with additive broadband noise. It is apparent that retaining the phase component is of utmost importance to the proper recontruction of the original image structure.

The importance of phase also extends to one-dimensional signals. It has been shown that the intelligibility of a speech sentence is retained if the inverse transform of the Fourier phase of a long segment of the speech signal is combined with unity magnitude to obtain the phase-only equivalent speech [24]. In fact, in listening to this processed sentence, total intelligibility is retained although the speech has the general quality associated with high-pass filtering and the introduction of additive white noise. The magnitude-only speech has some structure which provides a speech-like characteristic but with no speech intelligibility.

\section{COLOR-BRIGHTNESS SEPARATION}

There are several advantages to the separation of color from brightness information in image processing. Perceptual experimental evidence has established that the human visual system has a much higher sensitivity to changes in brightness details than to color. Moreover, there seems to be general agreement that spatial resolution is markedly lower in the chromatic channels, as is clear from figure $3(a, b)$, than in the achromatic one, as in figure 3 (L). Hence, high frequency information, representing fine details and edges, come mainly from the achromatic channel [36], [14]. This consideration and experimental results suggest that a color model which separates luminance from chrominance is most suitable for our image compression framework, where we can take advantage of the inherent lower spatial information carried in the color channels to embed them at a reduced-size image resolution inside the brightness information without altering the fine details in the original image.

Several color space representations exist that separate an RGB color image into separate brightness and color components. For instance, HSV space and HLS space are transformations of RGB space that can describe colors in terms more natural to an artist. The name HSV stands for hue, saturation, and value, and HLS stands for hue, lightness, and saturation, where the value or lightness components represent the luminance channel, and the hue/saturation components are the chromatic channels. The only disadvantage of the HSV and HLS spaces are the fact that they are device-dependent where one imaging sensor may produce a different color value from another sensor depending on how it is manufactured.

Some color spaces can express color in a deviceindependent way. Whereas HSV colors vary with imaging sensor hardware characteristics, device-independent colors are meant to be true representations of colors as perceived by the human eye. These color representations, called deviceindependent color spaces, are the result of work carried out in 1931 by the Commission Internationale d'Eclairage (CIE) and for that reason are also called CIE-based color spaces. The CIE created a set of color spaces that specify color in terms of human perception. It then developed algorithms to derive three imaginary primary constituents of color, that can be combined at different levels, to produce all the colors the human eye can perceive. The resulting CIE color models form the basis for all color management systems.

Although the RGB and HSV color representations differ from device to device, human perception of color remains consistent across devices. One such consistent color representation is the CIE L*a*b* color space, which is a nonlinear transformation of the RGB color space, that specifies color in terms of human perception in a way that is independent of the characteristics of any particular imaging device. We, thus, choose to separate the high resolution RGB color image into separate luminace and chrominance channels using the CIE L*a*b* color space ${ }^{2}$, where $\mathrm{L}^{*}$ represents luminance (brightness) image values, $\mathrm{a}^{*}$ represents redness-greenness, and $b^{*}$ represents yellowness-blueness color values [13].

\section{The Proposed LqSteg Compression Scheme}

The $\mathrm{L} * \mathrm{a} * \mathrm{~b} *$ color space separates a digitally acquired RGB image into a luminance channel $L$, and two chrominance channels $(a, b)$. In general the luminance channel $(L)$ suffers less noise artifacts than the $(a, b)$ chrominance channels, as well as retaining all the high-quality fine details of the original color image [31]. These considerations prompt us to embed reducedsize thumbnails of the $(\mathrm{R}, \mathrm{G}, \mathrm{B})$ color components of the original image inside the (less-important) Fourier magnitude spectrum of the full-size luminance channel, while maintaining the phase spectrum intact to avoide any modifications to the original fine-detailed information in the image.

The proposed technique we describe in this section introduces quality improvements over results obtained using the preliminary idea first presented in [32]. The former method suffered from discoloration artifacts due to the color embedding scheme adopted which did not deal with contaminating noise added to the RGB thumbnail images during the extraction process. This old technique also suffered from RGB embeddings being placed too close to the borders of the significant frequency coefficients in the Fourier spectral magnitude of the luminance channel of the source image, which resulted in further noise added to the $(\mathrm{R}, \mathrm{G}, \mathrm{B})$ components during extraction. These types of additive noise caused color artifacts

\footnotetext{
${ }^{2}$ For detailed information about the CIE color spaces please visit their website at http://www.cie.co.at
} 


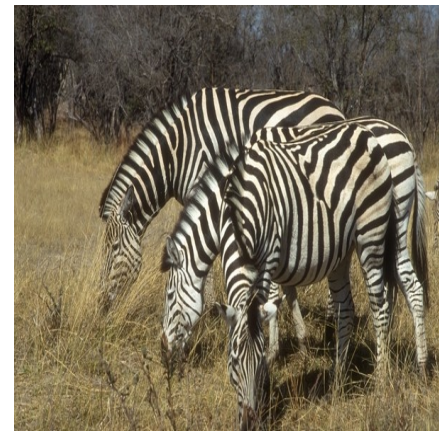

(a)

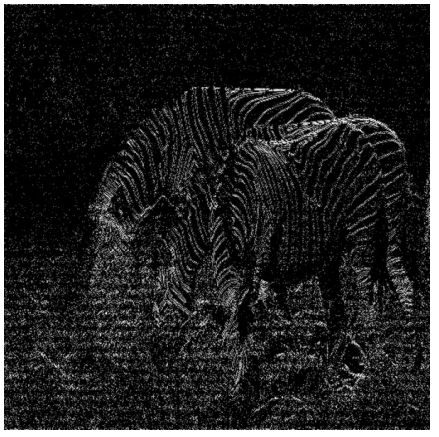

(b)

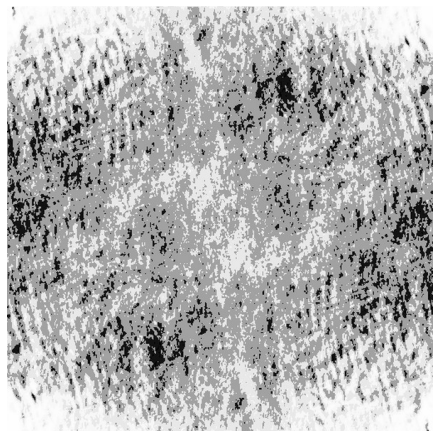

(c)

Fig. 2: (a) Zebras image, (b) Inverse Fourier transform of $e^{j \theta(u, v)}$ (the phase-only image of the Red-channel of the Zebras image). (c) Inverse Fourier transform of $|F(u, v)|$ (the magnitude-only image of the Red-channel of the Zebras image).
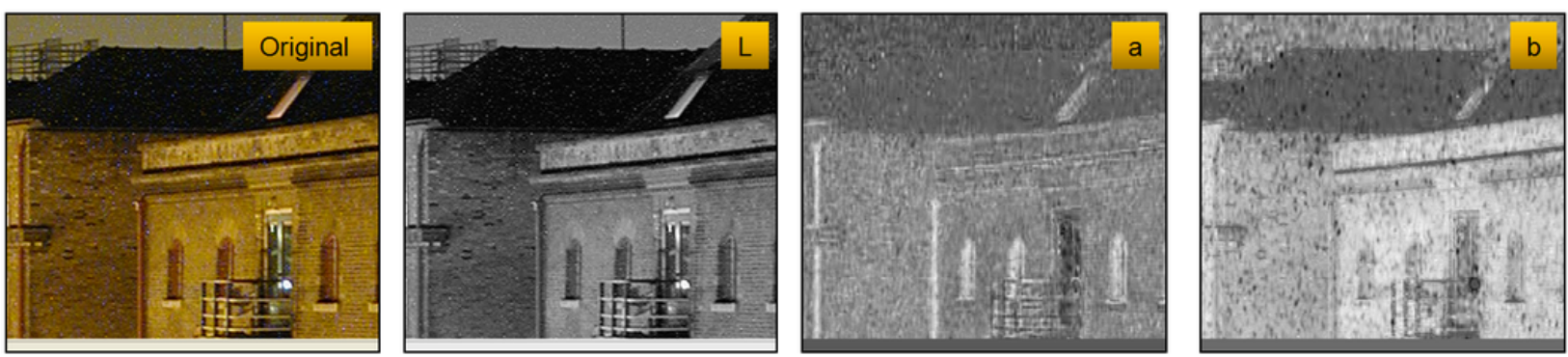

Fig. 3: A color image of a House separated into its component channels using the L*a*b* color space to show that spatial resolution is markedly lower in the chromatic channels $(a)$ and $(b)$ than in the achromatic luminance $(L)$ channel.

when regenerating the decompressed color image as will be shown from figure 7 when we talk about the experimental results in section VI. The comparisons shown in figure 7 are between example results obtained using the method in [32] and the LqSteg scheme we describe in this work. The figure shows the extracted RGB components using both methods and it is clear how high-frequency noise contaminates the extracted RGB components when using the old method.

This noise was however dealt with in the old scheme by applying a wavelet denoising operator (the default wdencmp Matlab wavelet denoising filter) on the extracted noisy RGB components which resulted in a smoothing effect on the color channels thus causing a faded color effect on the decompressed recovered image as shown in the image of figure 7(c). The fine details were however preserved in the unaltered luminance component. This denoising step has been rendered mostly unnecessary in our new LqSteg implementation, which results in major improvements in reproduction of colors in the decompressed image, as well as a boost in the speed of the decompression process.

\section{A. Compression: Embedding Chrominance into Luminance}

The 2D Fourier transform of the luminance channel is first computed and the magnitude spectrum is separated from the phase spectrum. Let $f_{L}(x, y)$ be the space-domain luminance channel of the acquired image $f(x, y)$. The Fourier transform of this luminance channel can be expressed in polar form as:

$$
F_{L}(u, v)=M_{L}(u, v) e^{j \theta_{L}(u, v)}
$$

where $M_{L}=\left|F_{L}(u, v)\right|$ is the luminance magnitude spectrum, $\theta_{L}(u, v)$ is its phase angle, and $(u, v)$ are the frequency coordinates.

The technique used to embed the reduced-size RGB color components into the Fourier magnitude of the luminance channel of the acquired image is to replace the low-amplitude-high frequency (insignificant) areas in this luminance magnitude spectrum with downsampled 1/8-size (thumbnail) (R,G,B) image components in a triangular formation (clearly depicted in figure 5). This type of embedding prevents aliasing of the color components when extracted (which appears as a mirroring of parts of the RGB component images from one side onto the opposite side and causes data loss).

The implications of embedding the RGB thumbnail images in the high frequency areas of the Fourier magnitude is a twofold effect; firstly, an additive noise component is introduced in the modified space-domain luminance image. The amplitude of this noise is proportionate to the variance in the RGB images. The smaller the changes in the RGB images, the less noise that appears in the luminance image, and vice versa. Secondly, an additive noise component is introduced in the extracted $(\mathrm{R}, \mathrm{G}, \mathrm{B})$ component images during the decompression process due to the Fourier-Inverse-Fourier transform operations which take place during embedding-extraction process, where the $(\mathrm{R}, \mathrm{G}, \mathrm{B})$ embedding location in the luminance magnitude spectrum is mostly where high-frequency noise resides.

Nevertheless, the two types of noise effects are dealt with in the implementation. The first type of additive noise is removed during the decompression stage of regenerating the full 


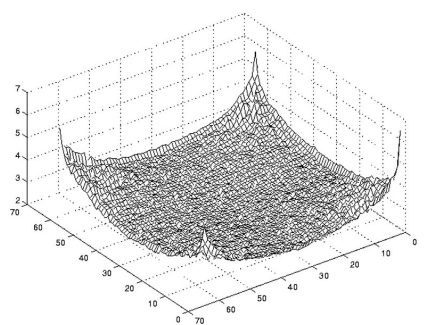

(a)

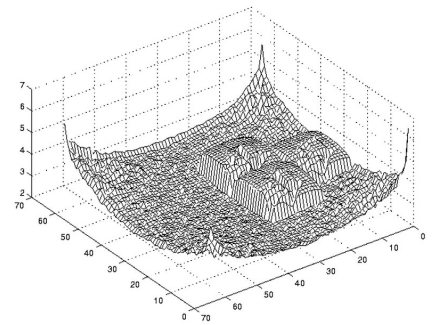

(b)
Fig. 4: (a) A typical Magnitude spectrum of the acquired image before embedding occurs, (b) The embedded (R,G,B) color components in the low-amplitude-high-frequency region of the Magnitude spectrum in a triangular formation.

color image by zero-padding the locations left behind in the magnitude spectrum of the luminance channel after extraction of the embedded thumbnail RGB component images. This zero-padding has the effect of suppressing any high frequency noise, thus preserving the quality of the original high-detail luminance image.

The second type of noise is avoided altogether by raising the gain of the pixel values of the embedded RGB thumbnail images before the embedding process. This is done by rescaling the pixel-intensity range for each individual $(R, G, B)$ component to be in the range $[0, k]$ instead of the normal range $[0,255]$, where $k$ is emperically set to a value greater than the maximum spectral noise amplitude measured inside the embedding locations of the spectral magnitude of the luminance image. This has the effect of raising the signal-tonoise ratio of the embedded RGB components, which results in high signal-to-noise ratio during extraction.

Figure 4 shows before and after figures of the luminance magnitude spectrum of a typical image when the reduced-size RGB components are embedded in the high frequency areas of this magnitude spectrum in a triangular formation.

This modified Fourier magnitude $\hat{M}_{L}(u, v)$ in which the RGB thumbnail images are embeded is then combined with the complex Fourier phase of the original luminance image to produce the modified Fourier spectrum of the luminance channel as in equation (3), which when transformed back to the space domain, using the inverse Fourier transform, will produce the space-domain luminance image which represents the compressed image ready for storage and transmission (as depicted in figure 5). This compressed luminance image is then stored in the JPEG format set to $100 \%$ compression quality This further improves the compression rate, as will be clear from table I, since it provides a two-stage compression; color compression inside the luminance image followed by JPEG compression of this compressed luminance image.

$$
\hat{F}_{L}(u, v)=\hat{M}_{L}(u, v) e^{j \theta_{L}(u, v)} .
$$

\section{B. Decompression: The Extraction Process}

Extraction of the hidden color information and recompositing (decompressing) the full color image takes place after com-

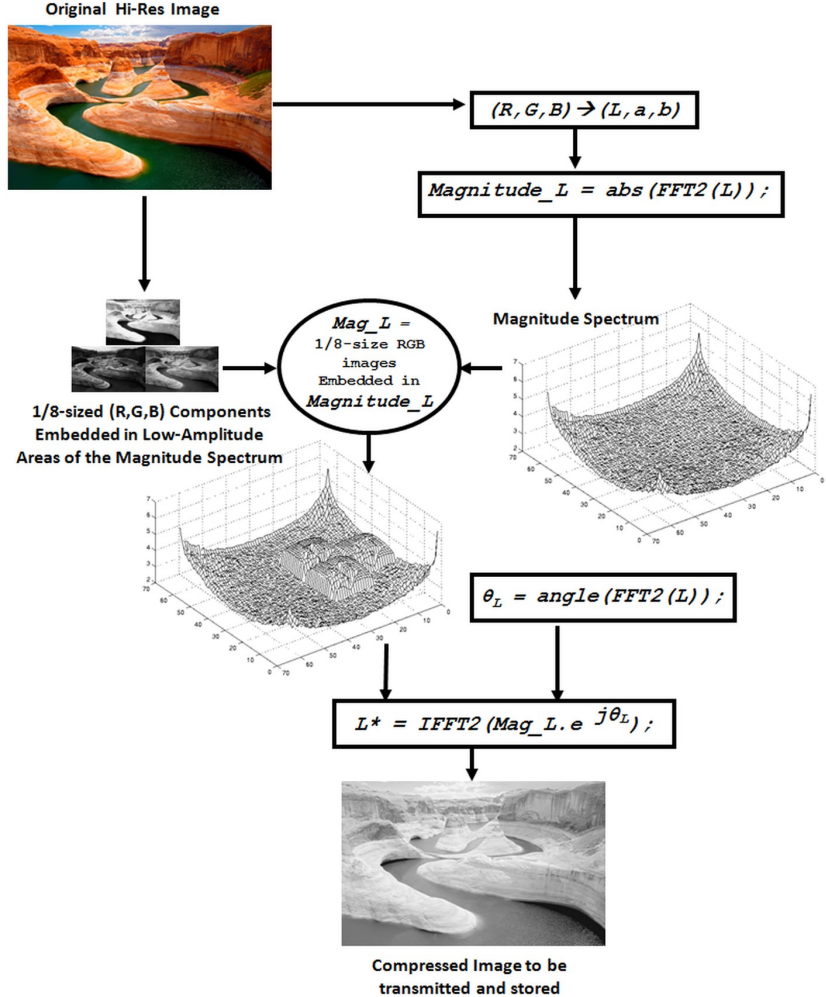

Fig. 5: Block diagram showing the general steps to embed the RGB color image thumbnails inside the Fourier magnitude of the Luminace channel after separating Luminance from Chrominance information.

puting the magnitude of the Fourier transform $\left(\hat{F}_{L}(u, v)\right)$ of the modified (compressed) luminance image to give: $\hat{M}_{L}(u, v)$.

The decompression steps are given as follows:

1) The reduced-sized RGB component images are extracted from their original locations inside this luminance magnitde spectrum $\hat{M}_{L}(u, v)$ and their pixel values are rescaled back to their original $[0,255]$ range.

2) The locations inside the luminance magnitde spectrum of extracted RGB thumbnail images are zeropadded to give a restored luminance magnitude spectrum given by: $\bar{M}_{L}(u, v)$. It is this magnitude spectrum which is then combined with the unaltered Fourier phase $e^{j \theta_{L}(u, v)}$ of the luminance image to produce the Fourier spectrum of the luminance channel as in equation (4), which when transformed back to the space domain, using the inverse Fourier transform, will produce the full-size space-domain luminance channel image $\bar{L}(x, y)$ which retains all the fine details of the original image.

$$
\bar{F}_{L}(u, v)=\bar{M}_{L}(u, v) e^{j \theta_{L}(u, v)} .
$$

3) The next step is to transform the low-resolution reduced-sized RGB thumbnail components to the $\mathrm{L} * \mathrm{a} * \mathrm{~b} *$ color space. The low-resolution $(L)$ component is then discarded. 
4) The $(\hat{a}, \hat{b})$ components are then resized using highquality interpolation to the same resolution size of the original image to give $(\bar{a}, \bar{b})$.

5) The resized $(\bar{a}, \bar{b})$ components are then combined with the full-size space-domain luminance channel image, generated from its component magnitude and phase spectra in step (2), to form the three components $(\bar{L}, \bar{a}, \bar{b})$.

6) This color image in the $\mathrm{L}^{*} \mathrm{a} \mathrm{b}^{*}$ space is then transformed back to the RGB space to produce the recovered decompressed high-resolution color image, which retains all the fine details that would otherwise be lost if other lossy compression techniques were used.

\section{EXPERIMENTAL RESULTS}

In this section, experimental results are presented to show the performance of our LqSteg image compression scheme when used to compress a high-resolution image into a luminance image, and regenerate the decompressed high-resolution color image back. Our LqSteg compression scheme described in previous sections was implemented in the Matlab programming environment.

\section{A. Image Quality Measures}

In evaluating the performance of our LqSteg compression scheme it is important to take into consideration both the analytical performance of the algorithm as well as the visual quality of the decompressed images generated by the scheme in comparison to the ideal image. The most important tests are related to our human perception, the ultimate measure of visual fidelity, which is very subjective.

The subjective tests are carried out by people who look for visual differences between the images (original and recovered image) trying to find which one of them is the original. If the percentage of success is below $50 \%$, it can be concluded that the quality of the recovered image is close enough to that of the original. The subjective test's rules and recommendations are defined by the International Telecommunication Union [16], [33].

Unlike the subjective approach which is based on human vision, the well known mean-square-error (MSE) metric calculates the global error variance (power in the difference image) between an ideal image $f$, and the recovered image $\hat{f}$, and has been widely used for measuring the performance of various filters [2]. The only shortcoming in an MSE metric is that it is not ideal for tracking visual quality in the estimated image, because it is sensitive to minor pixel variations between the ideal and recovered images that do not, in general, affect the perceived visual quality.

A more robust measure of decompression performance that has been widely used by the signal processing community is the Peak-Signal-to-Noise-Ratio (PSNR) in decibels (dB) [9], [26], given by:

$$
\operatorname{PSNR}=20 \log _{10}\left(\frac{L-1}{\sigma_{e}}\right)
$$
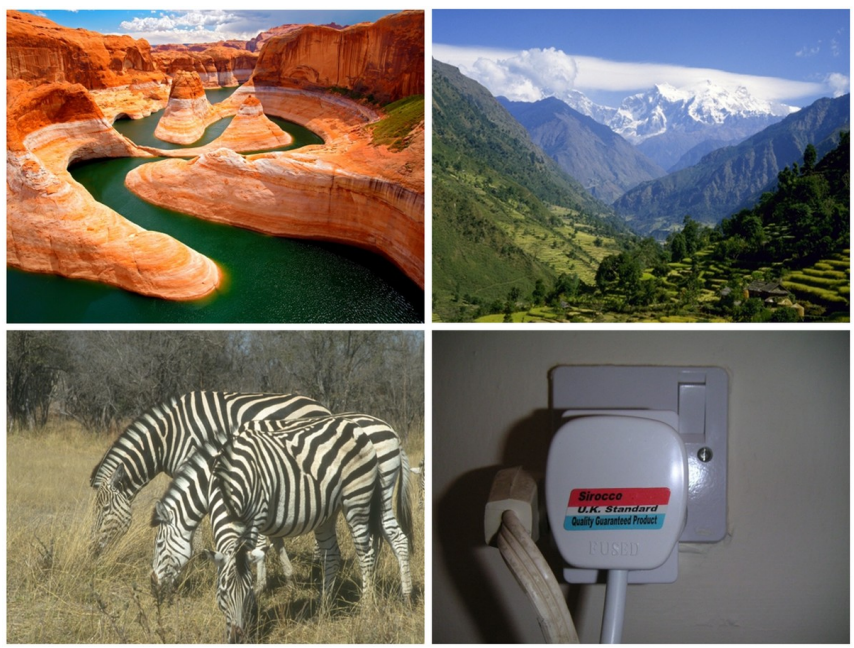

Fig. 6: Four mega-pixel-size color test images (top left and right: Lake, Mountain, bottom left and right: Zebras, Plug) which represent images of various detail and color structure.

where $L$ is the number of gray levels in an image ( $L=256$ for 8-bit images), and $\sigma_{e}$ is the residual standard deviation in the error image given as:

$$
\sigma_{e}=\sqrt{\frac{1}{S} \sum_{s=0}^{S-1}\left(f_{s}-\hat{f}_{s}\right)^{2}}
$$

for $S$-sized images.

This PSNR metric is an engineering term for the ratio between the maximum possible power of a signal and the power of corrupting noise that affects the fidelity of its representation. The PSNR is most commonly used to measure the quality of reconstruction in an image by comparing the decompressed image with the original image. This measure is less sensitive to minor deviations between images and will be adopted for comparing our results.

It is important to note, however, that objective measures such as the PSNR and MSE metrics are not necessarily correlated to our perception of an image. This is because methods that are least squares based are optimum in terms of MSE values without necessarily producing the best visual results.

\section{B. Results of Proposed Method}

We start by showing the original test images used in our experiments. Figure 6 shows four mega-pixel-size color test images (top left and right: Lake, Mountain, bottom left and right: Zebras, Plug) which represent images of various detail and color structure.

The comparisons shown in figure 7 are between results obtained using the method in [32] and our LqSteg scheme described in this work. The figure shows the extracted RGB components using both methods and it is clear how highfrequency noise contaminates the extracted RGB components when using the old method. It also shows how our technique reproduces the colors in the original image to a high degree of accuracy whithout the loss of any fine details. 


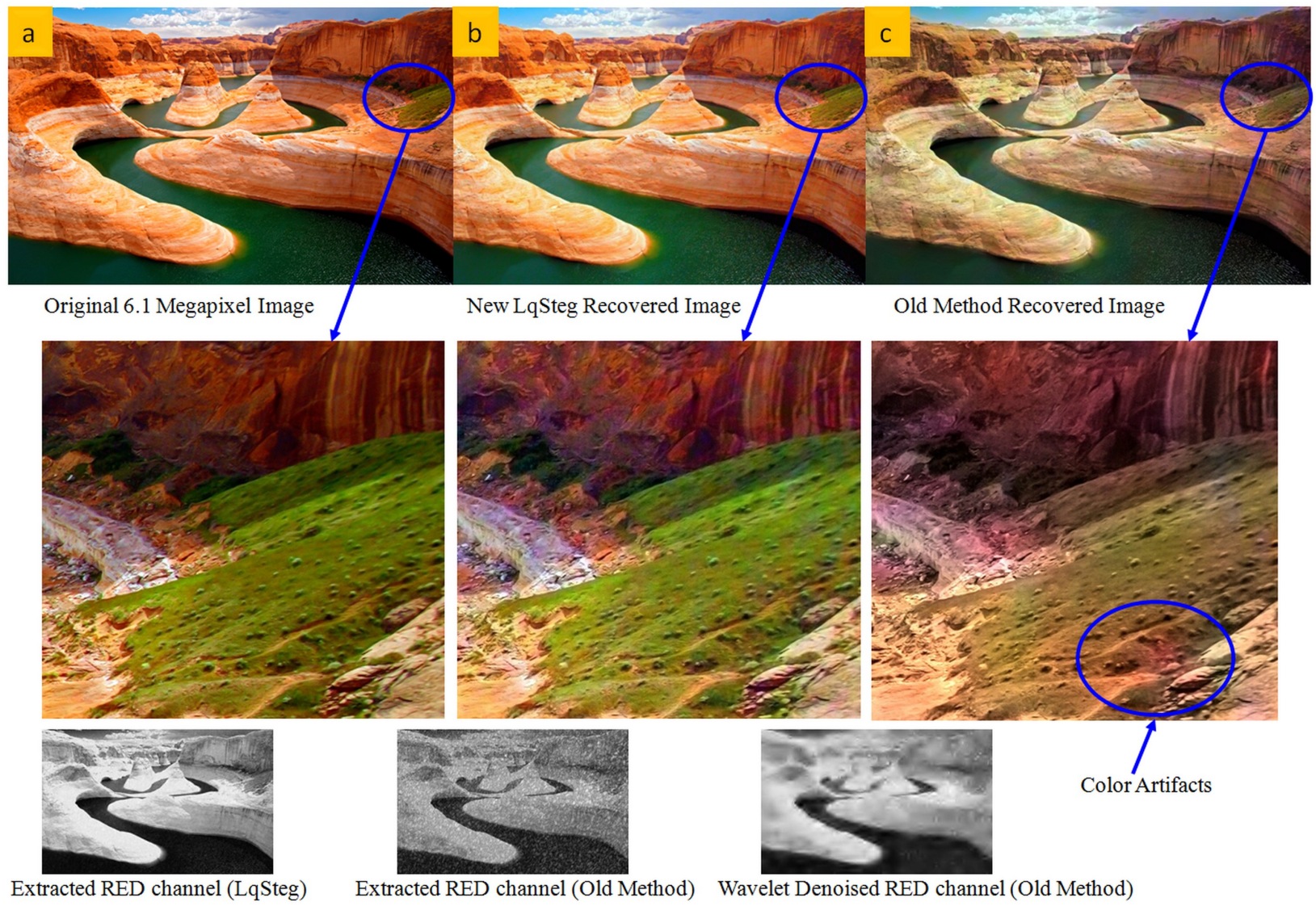

Fig. 7: Comparison between results obtained using the method in [32] and our new LqSteg method described in this work.

In figure 8 we show compression results for the Zebras image at 8 megapixels. The left image shows the original Zebras image, the middle image shows the compressed Luminance image with a compression rate at $86.20 \%$, and the right image shows the decompressed image where the colors have been accurately reproduced and all the fine details maintained.

Next, in figure 9-(a) we show a RAW color image (with no compression of any sort) of a mountain scene acquired by a digital camera using a high 3.9 megapixel resolution of $2560 \times 1600$ (3.9 mega pixels) and 12 megabytes (MB) in size. Figure 9-(b) shows the compressed image using our Fourierstego-based compression technique (LqSteg) with the RGB color thumbnail images embedded in the luminance image. The size was reduced to $1.6 \mathrm{MB}$ (i.e. an $86.29 \%$ compression rate was achieved). The regenerated high-resolution color image is shown in figure 9-(c) where it is clear that the colors were preserved in the spectral magnitude of the luminance channel and recovered properly.

The PSNR between the individual RGB color channels of the original raw color image and the decompressed color image using our stego-compression scheme was $P S N R_{R}=32.14$ $\mathrm{dB}, P S N R_{G}=39.10 \mathrm{~dB}, P S N R_{B}=27.79 \mathrm{~dB}$, and the average $P S N R_{a v}=33.02 \mathrm{~dB}$

Next, we present results of compressing a color image acquired using a mega-pixel camera. Figure 10-(a) shows the original RAW color image of size $1280 \times 960$ pixels $(1.17$ mega pixels) and file size of 3.6 MB. Figure 10-(b) shows the compressed image where the file size was reduced to $625 \mathrm{kB}$ (i.e. $82.64 \%$ compression rate). In comparison, the compression rate of the JPEG standard set to $100 \%$ quality for the same image in figure 10-(a) was $77.78 \%$. Also, the maximum compression rate that Zhang \& Zhang were able to achieve in [39] for grey-scale image compression was $1 / 2$ of the original image file size (i.e. $50 \%$ compression rate), while Kang et. al in [18] were able to improve the JPEG compression rate by adding an extra $0.36 \%$ to a current rate.

The PSNR between the individual RGB color channels of the original raw color image and the decompressed color image was $P S N R_{R}=25.22 \mathrm{~dB}, P S N R_{G}=31.60 \mathrm{~dB}$, $P S N R_{B}=26.62 \mathrm{~dB}$, and the average $P S N R_{a v}=27.81 \mathrm{~dB}$. It should be noted that the PSNR of the grey-scale compression scheme of Zhang \& Zhang [39] was generally higher than our scheme at $36 \mathrm{~dB}$, but as previously mentioned, at much lower compression rates. Figure 10-(c) shows the regenerated highresolution color image where it is clear that the colors were preserved in the spectral magnitude of the luminance channel and recovered properly.

Table I clearly shows how our LqSteg compression scheme exceeds the compression rates for high-quality JPEG compressions for all image sizes, while staying comparable in terms of PSNR. One observation when comparing the images in Figure 9-(b) and Figure 10-(b) as well as tracking the compression rates from table $\mathrm{I}$ is that the compression rates increase as the size of the test images increase. 

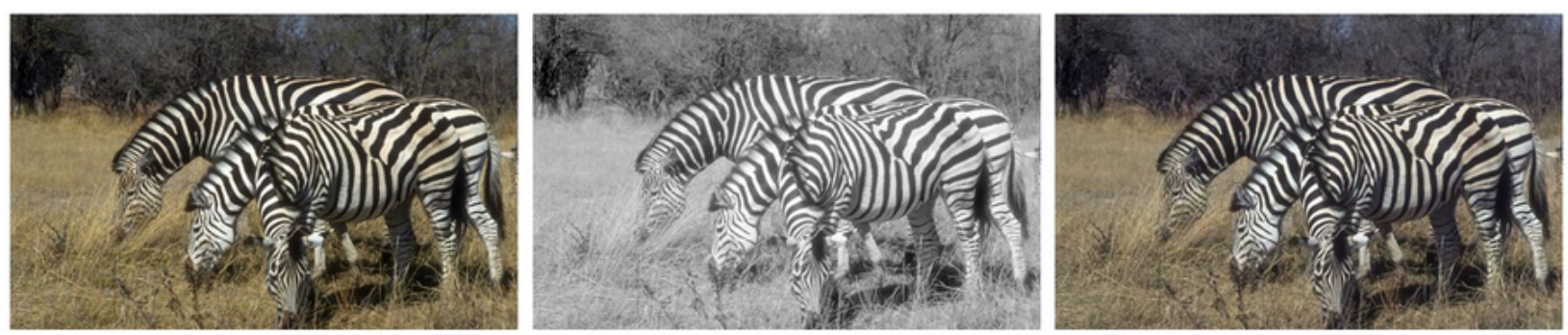

Fig. 8: Compression results for the Zebras image at 8 megapixels. The left image shows the original Zebras image, the middle image shows the compressed Luminance image with a compression rate at $86.20 \%$, and the right image shows the decompressed image where the colors have been accurately reproduced and all the fine details maintained.

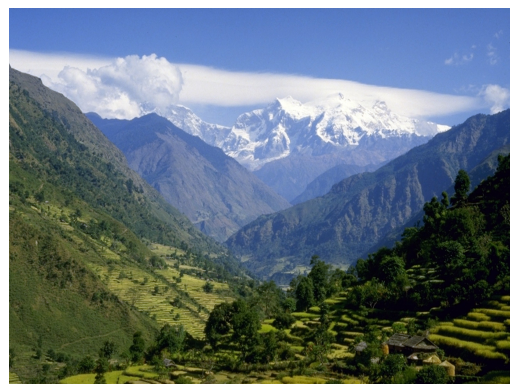

(a)

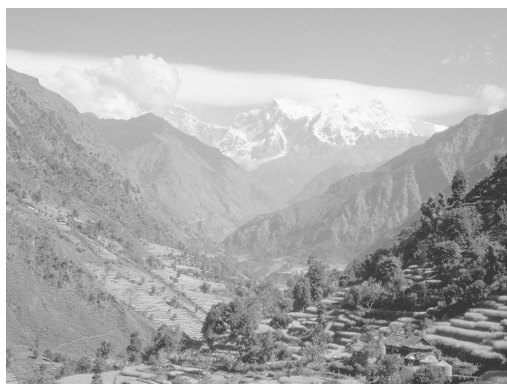

(b)

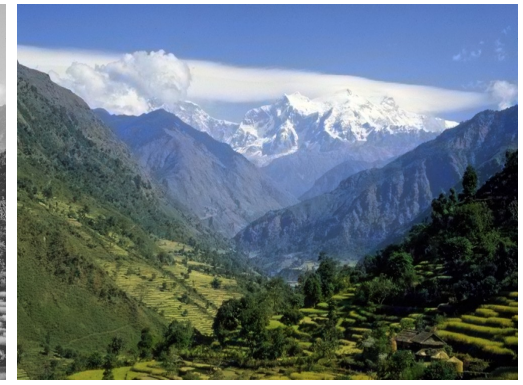

(c)

Fig. 9: (a) Original mountain scene of size $2560 \times 1600$ pixels (3.9 mega pixels) and a file size of $12 \mathrm{MB}$, (b) Compressed image with RGB thumbnail images embedded in the spectral magnitude of the luminance channel, with file size reduce to $1.6 \mathrm{MB}$ equivalent to a compression rate of $86.29 \%$. (c) Recovered high-resolution image retaining the original high-resolution quality and colors.

TABLE I: Compression Rate for our Lossless-quality Stego compression scheme (LqSteg) in comparison to the Compression Rate of the JPEG standard set to $100 \%$ Quality. The table also shows the average PSNR (dB) achieved for each method at various image megapixel sizes.

\begin{tabular}{|l||c||c|c||c|c|}
\hline Image & Images Size (MegaPixels) & LqSteg Compression Rate (\%) & LqSteg $P S N R_{a v}(\mathrm{~dB})$ & JPEG Compression Rate (\%) & JPEG PSNR $R_{a v}(\mathrm{~dB})$ \\
\hline \hline \multirow{3}{*}{ Mountain } & 0.30 & 78.47 & 30.84 & 73.14 & 37.98 \\
\cline { 2 - 6 } & 1.17 & 83.42 & 32.19 & 80.37 & 39.08 \\
\cline { 2 - 6 } & 3.90 & 86.29 & 33.02 & 83.80 & 39.45 \\
\cline { 2 - 6 } & 8.00 & 87.99 & 33.06 & 85.37 & 39.50 \\
\hline \hline \multirow{3}{*}{ Zebras } & 0.30 & 72.59 & 31.56 & 68.15 & 37.39 \\
& 1.17 & 79.34 & 33.05 & 75.65 & 38.71 \\
\cline { 2 - 6 } & 3.90 & 83.84 & 33.45 & 83.08 & 39.27 \\
\cline { 2 - 6 } & 8.00 & 86.20 & 26.04 & 72.56 & 32.37 \\
\hline \hline Lake & 6.10 & 80.69 & 27.81 & 77.78 & 35.79 \\
\hline Plug & 1.17 & 82.64 & & & \\
\hline
\end{tabular}

Finally, figure 11 shows the compression rates in $\%$ and the decompressed maximum achievable PSNR in $\mathrm{dB}$ plotted against various image sizes (in mega-pixels) for the Mountain and Zebras test color images. It clearly shows that both PSNR and compression rates increase slightly as the image size increases.

\section{CONCLUSION}

This paper has developed the LqSteg high-quality color image compression framework, that demonstrated significant improvements over the Fourier-domain image compression paradigm introduced in [32] and in [30]. This new steganography-based compression scheme utilized spacedomain and frequency-domain color image processing operations that addressed both issues of quality and reduced storage size. Experimental results as well as objective image quality measures have shown that our technique is very competitive with the widely used JPEG lossy image compression standard with the added advantage of being able to recover the original quality without blocking artifact degradations or loss of highfrequency details common in lossy compression techniques. In future work, we would like to investigate using the discrete cosine transform (DCT) domain instead of the fast fourier transform domain. The DCT possesses a high energy compaction property that would allow for an increase in the size 


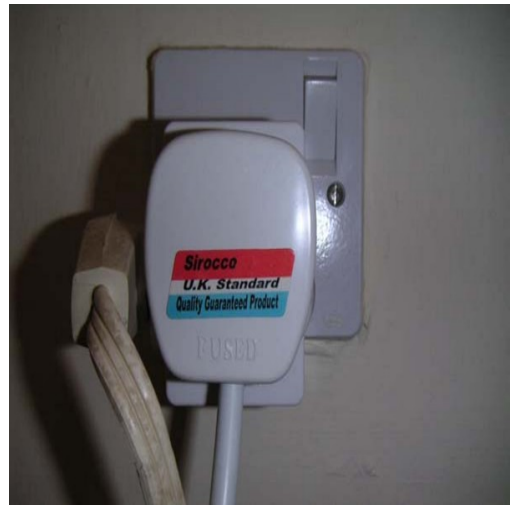

(a)

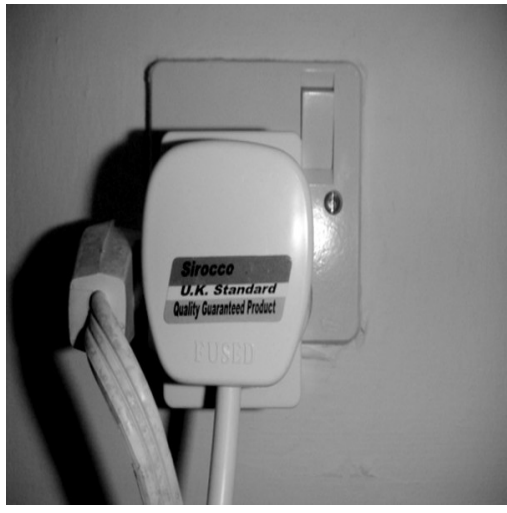

(b)

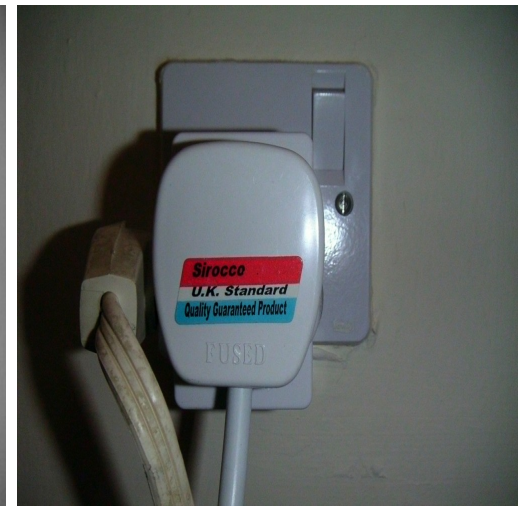

(c)

Fig. 10: (a) Original Plug image of size $1280 \times 960$ pixels (1.17 mega pixels) and file size of $3.6 \mathrm{MB}$, (b) Compressed image with RGB thumbnail images embedded in the spectral magnitude of the luminance channel, file size was reduced to $625 \mathrm{kB}$ (i.e. $82.64 \%$ compression rate). (c) Recovered high-resolution image, retaining the original high-resolution quality and colors.

of the embedded RGB color components which should further improve the quality of the regenerated decompressed color image.

\section{ACKNOWLEDGMENT}

The author would like to thank the anonymous reviewers for their valuable comments that helped improve the original manuscript.

\section{REFERENCES}

[1] Patrizio Campisi, Deepa Kundur, Dimitrios Hatzinakos, and Alessandro Neri. Compressive data hiding: An unconventional approach for improved color image coding. EURASIP Journal on Applied Signal Processing, 2002(1):152-163, 2002.

[2] K.R. Castleman. Digital Image Processing. Prentice Hall, Upper Saddle, NJ, 1996

[3] M. U. Celik, G. Sharma, A. M. Tekalp, and E. Saber. Lossless generalized-lsb data embedding. IEEE Trans. Image Processing, 14(2):253-266, Feb. 2005

[4] Chi Kwong Chan and L.M. Cheng. Hiding data in images by simple LSB substitution. Pattern Recognition, 37:469-474, 2004.

[5] C. C. Chang, Y. H. Chen, and C. C. Lin. A data embedding scheme for color images based on genetic algorithm and absolute moment block truncation coding. Soft Computing, 13:21-331, October 2008.

[6] C. C. Chang, J. Y. Hsiao, and C. S. Chan. Finding optimal leastsignificant-bit substitution in image hiding by dynamic programming strategy. Pattern Recognition, 36(7):1595-1683, July 2003.

[7] C. C. Chang, C. C. Lin, C. S. Tseng, and W. L. Tai. Reversible hiding in dct-based compressed images. Information Sciences, 177:2768-2786, July 2007.

[8] C. C. Chang, W. L. Tai, and C. C. Lin. A reversible data hiding scheme based on side match vector quantization. IEEE Trans. Circuits and Systems for Video Technology, 16(10):1301-1308, 2006.

[9] Chin-Chen Chang, Tung-Shou Chen, and Lou-Zo Chung. A steganographic method based upon jpeg and quantization table modification. Information Sciences, 141(1):123-138, 2002.

[10] Marc Chaumont and William Puech. A dct-based data-hiding method to embed the color information in a jpeg grey level image. In $E U$ SIPCO'06: The European Signal Processing Conference, page 5, 2006.

[11] B. Chen and G.W. Wornell. Quantization index modulation: A class of provably good methods for digital watermarking and information embedding. IEEE Trans. Information Theory, 47(4):1423-1443, 2001.
[12] Kevin Curran and Karen Bailey. An evaluation of image based steganography methods. International Journal of Digital Evidence, 2(2):1-40, Fall 2003.

[13] Mark D Fairchild. Color appearance models. John Wiley \& Sons, 2013.

[14] Per-Erik Forssen, Gosta Granlund, and Johan Wiklund. Channel representation of colour images. Technical Report LiTH-ISY-R-2418, Computer Vision Laboratory, Department of Electrical Engineering, Linkoping University, SE-581 83 Linkoping, Sweden, March 2002.

[15] T.S. Huang, J.W. Burnett, and A.D. Deczky. The importance of phase in image processing filters. IEEE Trans. on ASSP, 23(6):529-542, 1975.

[16] ISO IEC. Information technology-digital compression and coding of continuous-tone still images: Requirements and guidelines. Standard, ISO IEC, pages 10918-1, 1994.

[17] A.K. Jain, U. Uludag, and R.L. Hsu. Hiding a face in a fingerprint image. In Proc. of the International Conference on Pattern Recognition (ICPR), Quebec City, Canada, August 2002.

[18] Sang-ug Kang, Xiaochao Qu, and Hyoung Joong Kim. Compressing JPEG compressed image using reversible data hiding technique. In Signal and Information Processing Association Annual Summit and Conference (APSIPA), 2013 Asia-Pacific, pages 1-7. IEEE, 2013.

[19] Ying Luo and Rabab Kreidieh Ward. Removing the blocking artifacts of block-based dct compressed images. Image Processing, IEEE Transactions on, 12(7):838-842, 2003.

[20] Lisa M. Marvel, Jr. Charles G. Boncelet, and Charles T. Retter. Spread spectrum image steganography. IEEE Trans. Image Processing, 8(8):1075-1083, August 1999.

[21] Krishnan Nallaperumal, Jennifer J Ranjani, Seldev Christopher, and S Saudia. Removal of blocking artifacts in jpeg compressed images using dual tree complex wavelet filters for multimedia on the web. In Wireless and Optical Communications Networks, 2006 IFIP International Conference on, pages 4-pp. IEEE, 2006.

[22] Aria Nosratinia. Enhancement of jpeg-compressed images by reapplication of jpeg. Journal of VLSI signal processing systems for signal, image and video technology, 27(1-2):69-79, 2001.

[23] Koichi Nozaki, Michiharu Niimi, Richard O. Eason, and Eiji Kawaguchi. A large capacity steganography using color bmp images. In ACCV '98: Proceedings of the Third Asian Conference on Computer Vision-Volume I, pages 112-119, London, UK, 1998. Springer-Verlag.

[24] A.V. Oppenheim and J.S. Lim. The importance of phase in signals. Proc. of the IEEE., 69(5):529-541, May 1981.

[25] A.V. Oppenheim, J.S. Lim, and S.R. Curtis. Signal synthesis and reconstruction from partial fourier-domain information. Journal of the Optical Society of America, 73(11):1413-1420, 1983.

[26] George Pavlidis, A Tsompanopoulos, Nikos Papamarkos, and Christodoulos Chamzas. Jpeg2000 over noisy communication channels 

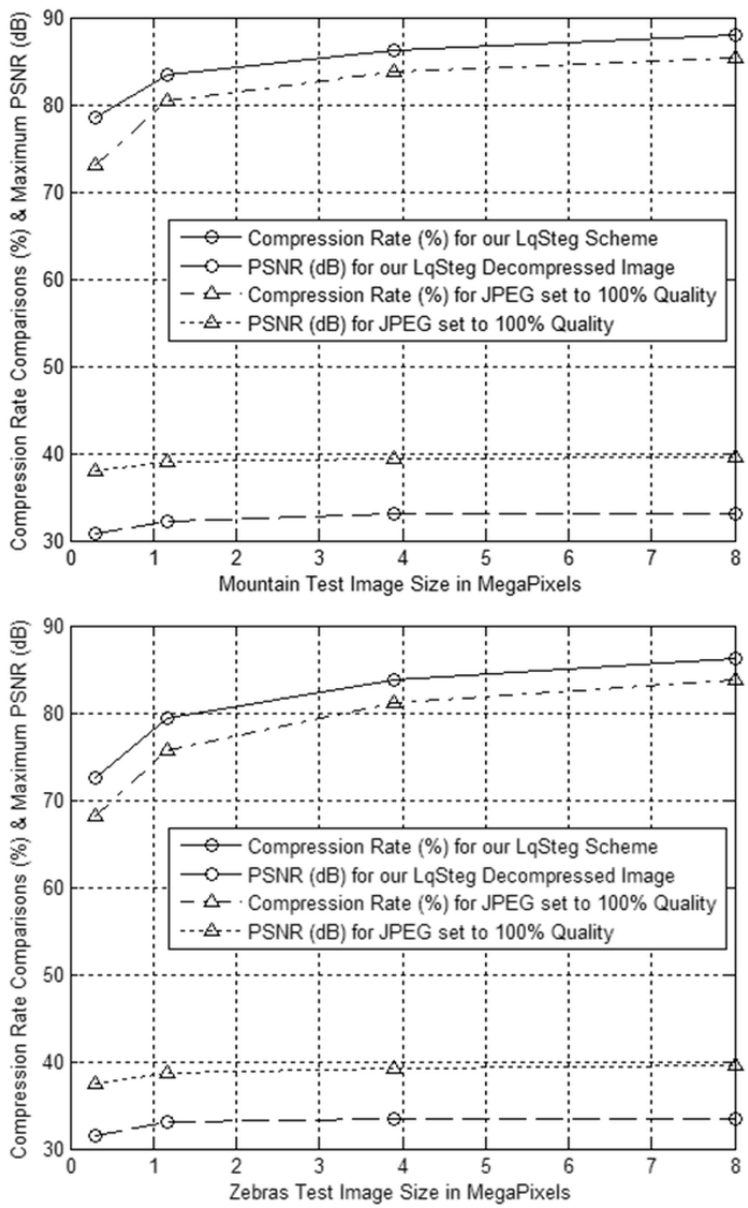

Fig. 11: Compression Rate for our LqSteg compression scheme in comparison to the compression rate for the JPEG standard set to $100 \%$ Quality. The plot also shows the maximum PSNR (dB) achieved by both methods for the recovered Mountain and Zebras images.

thorough evaluation and cost analysis. Signal Processing: Image Com- munication, 18(6):497-514, 2003.

[27] Fabien AP Petitcolas, Ross J Anderson, and Markus G Kuhn. Information hiding-A survey. Proceedings of the IEEE, 87(7):1062-1078, 1999.

[28] Niels Provos and Peter Honeyman. Hide and seek: An introduction to steganography. In IEEE Security \& Privacy Magazine, pages 32-44. IEEE Computer Society, 2003.

[29] Chuan Qin, Chin-Chen Chang, and Yi-Ping Chiu. A novel joint datahiding and compression scheme based on SMVQ and image inpainting. Image Processing, IEEE Transactions on, 23(3):969-978, 2014.

[30] T. Rabie. Frequency-domain data hiding based on the matryoshka principle. Special Issue on Advances in Video Processing and Security Analysis for Multimedia Communications, International Journal of Advanced Media and Communication, 1(3):298-312, 2007.

[31] Tamer Rabie. Adaptive hybrid mean and median filtering of high-iso long-exposure sensor noise for digital photography. SPIE Journal of Electronic Imaging, 13(2):264-277, April 2004.

[32] Tamer Rabie. A novel compression technique for super resolution color photography. In Innovations in Information Technology, 2006, pages 1-5. IEEE, 2006.

[33] JM Rodrigues, JR Rios, William Puech, et al. Ssb-4 system of steganography using bit 4. In 5th International Workshop on Image Analysis for Multimedia Interactive Services, 2004.

[34] Sukhwinder Singh, Vinod Kumar, and HK Verma. Reduction of blocking artifacts in jpeg compressed images. Digital Signal Processing, 17(1):225-243, 2007.

[35] Kaushal Solanki, Noah Jacobsen, Upamanyu Madhow, B. S. Manjunath, and Shivkumar Chandrasekaran. Robust image-adaptive data hiding using erasure and error correction. IEEE Trans. Image Processing, 13(12):1627-1639, December 2004.

[36] A.B. Watson. Perceptual-components architecture for digital video. Journal of the Optical Society of America A,, 7(10):1943-1954, 1990.

[37] Min Wu and Bede Liu. Data hiding in image and video:part I - fundamental issues and solutions. IEEE Trans. Image Processing, 12(6):685695, June 2003.

[38] Zixiang Xiong, Michael T Orchard, and Ya-Qin Zhang. A deblocking algorithm for jpeg compressed images using overcomplete wavelet representations. Circuits and Systems for Video Technology, IEEE Transactions on, 7(2):433-437, 1997.

[39] Xinpeng Zhang and Weiming Zhang. Semantic image compression based on data hiding. IET Image Processing, 9(1):54-61, 2014.

[40] Yang Zhao, Patrizio Campisi, and Deepa Kundur. Dual domain watermarking for authentication and compression of cultural heritage images. Image Processing, IEEE Transactions on, 13(3):430-448, 2004. 